\title{
Fabrication and characterization of dye-doped polymer optical fiber as a light amplifier
}

\author{
Mandamparambil Rajesh, Mavila Sheeba, Karinjamanna Geetha, Chakkalakkal P. G. Vallaban, \\ Padmanabhan Radhakrishnan, and Vadakkedathu P. N. Nampoori
}

\begin{abstract}
The fabrication and characterization of a Rhodamine 6G-doped polymer optical fiber amplifier have bet carried out. Two different schemes were employed to characterize the optical fiber: the stripe illumination technique to study the fiber as a gain medium and another technique to study its performance as amplifier. We observed a spectral narrowing from 42 to $7 \mathrm{~nm}$ when the pump energy was increased to $6 \mathrm{~mJ}$ in the stripe illumination geometry. A gain of $18 \mathrm{~dB}$ was obtained in the amplifier configuration. The effects of pump power and dye concentration on the performance of the fiber as an amplifier were also studied. 2007 Optical Saciety of America

OCIS codes: $\quad 060.0060,060.2320,060.2310,060.2280$.
\end{abstract}

\section{Introduction}

Polymer-based waveguide structures and devices have been found to be suitable for short distance communications and optical integrated circuits. Even though optical fiber networks are mainly focused on silica optical fibers (SOFs), polymer-optical-fiber (POF) -based systems are seriously being considered for short-distance communication. This is due to the competitiveness of POF in handling, flexibility, and cost effectiveness with respect to silica fibers., $1,2 \mathrm{Al}$ though a higher loss factor is a major handicap for POF, recently developed techniques for decreasing losses in poly(methyl methacrylate) (PMMA) -based POF have raised much interest in this field. ${ }^{3-7}$ The availability of inexpensive sources in the visible region has increased the utilization of POF in data communication over local area network (LAN) systems. ${ }^{8}$ The implementation of optical communication in the visible region demands the development of suitable optical amplifiers working in this region. ${ }^{9-17}$

The authors are with Cochin University of Science and Technol ogy, Cochin 682 022, India. M. Rajesh (rajeshmocusat.ac.in), M. Sheeba, K. Geetha, P. Radhakrishnan, and V. P. N. Nampoori (vpnnampoorigcusat.ac.in) are with the International School of Photonics. C. P. G. Vallaban, as well as M. Rajesh, is with the Center of Excellence in Lasers and Opto-Electronic Sciences.

Received 20 April 2006; revised 26 August 2006; accepted 30 August 2006; posted 13 September 2006 (Doc. ID 70162); published 15 December 2006.

$0003-6935 / 07 / 010106-07 \$ 15.00 / 0$

(C) 2007 Optical Society of America
POFs that are doped with dyes or rare-earth ele ments are potential candidates for this purpose.

Laser dyes, which act as highly efficient material for lasing and amplification, have a wide range of tunability in the visible region. ${ }^{10-15}$ The range of tunability of laser dyes such as Rhodamine $B$ and Rhor damine $6 \mathrm{G}$ usually lies between 570 and $640 \mathrm{~nm}$. The advantage of incorporating laser dyes in solid matrices such as POF is that it is easier and safer to handle them than when they are in liquid form. From recent studies it has been found that the dye-doped pols. mer materials have better efficiency, beam quality, and superior optical homogeneity when compared to other solid matrices. Here we focus our studies on the line narrowing behavior and the amplification char. acterization of fluorescence in Rhodamine 6G-doped polymer optical fibers.

\section{Fabrication of Dye-Doped Polymer Optical Fiber}

The base material used for the fabrication of polymer preforms is methyl methacrylate (MMA) monomer. ${ }^{\text {p-1 }}$ MMA is a suitable candidate for the fabrication of poly. mer preforms since it has good optical quality and is compatible with most of the organic dopants. The fractive index of pure MMA is approximately 1.41 , and it will increase up to 1.49 owing to volume reduction during the phase transition from liquid to solid We have concentrated primarily on only core fibers (air cladding), neglecting the scattering losses associated with it because of the absence of cladding.

Commercially available MMA will contain inhibitors such as hydroquinons. Inhibitors are used to transport MMA without polymerization. Inhibitors 
in removed by repeatedly washing the monomer th $5 \% \mathrm{NaOH}$ solution followed by flushing with witled water. The remaining water is removed by thing suitable drying agents such as $\mathrm{CaCl}_{2}$. The momer is purified by distillation under reduced hessure.

Suitable initiators such as benzoyl peroxide or azowisobutyronitrile (AIBN) are used to start the polyorization. We used benzoyl peroxide for our process nere is no gas such as nitrogen that gets reased during polymerization as in the case of AIBN. his reduces the possibility of air bubble formation the polymer preforms. Along with the initiator, butyl mercaptan is used as the chain transfer agent regulate and terminate the polymerization proxs. Adding an appropriate quantity of chain transogent and initiator controls the molecular weight it the monomer. The regulation of the molecular pright is an important factor that governs the drawwity of the polymer perform. An optimum molecuiI weight (typically between 60,000 and $10^{5}$ ) is fixed the number of trials. We have used Rhodamine 6G in $^{i-17}$ for our current study since it has relatively rod photostability and high fluorescence efficiency. hodamine $6 \mathrm{G}$ at 460 parts per million (ppm) conmatration is chosen for this study, and it is added bong with the initiator and chain transfer agent into se monomer. The resulting mixture is stirred well to mid aggregate formations.

The monomer mixed with the initiator, dye, and he chain transfer agent is poured into a glass test whe of the required diameter and length. The unimixing of the dye-monomer mixture was enmed by stirring well with a magnetic stirrer and ter filtered using filter paper to ensure that no unssolved part remained. This was then kept in a nastant temperature bath at $70^{\circ} \mathrm{C}$ for $48 \mathrm{~h}$, at $90^{\circ} \mathrm{C}$ $818 \mathrm{~h}$, and at $105^{\circ} \mathrm{C}$ in an air furnace for $8 \mathrm{~h}$. These leps led to a high quality polymer preform that could tused for drawing the fiber.

The prepared preform was then ready for drawing be fiber in a custom-made fiber drawing tower. By sing a preform feeder, the preform was lowered into furnace that was maintained at a stable temperahere of $180^{\circ} \mathrm{C}$, and fiber was drawn at this temperture. Optical fibers of various diameters were drawn 5 adjusting the feed rate of the preform and the haw rate of the fiber. Using the diffraction technique thomogeneity in diameter of the fiber was tested.

\section{Characterization of Dye-Doped Optical Fiber as Sht Amplifier}

the current use of optical fiber amplifier is based on titum-doped silica fibers working in the IR region. he recent introduction of polymer optical fibers in bort-distance communication and network designs wh as LANs has brought amplifiers and repeaters sing polymer optical fibers to the forefront of rewarch and development activities. The possibility of large bandwidth available in visible wavelengthlased data communication has encouraged studies eploring the possibility of using dye-doped optical fiber as an optical amplifier. ${ }^{9-13.18}$ The details of the work done in this area in our laboratory are given in the following sections.

\section{Experimental Setup for the Characterization of Dye-Doped Polymer Optical Fiber as a Gain Medium and as Optical Amplifier}

\section{A. Gain Medium}

A schematic of the experimental setup to characterize the dye-doped polymer optical fiber as an optical gain medium is given in Fig. 1. The main component of the experimental setup is the Rhodamine 6G-doped polymer $5 \mathrm{~cm}$ long optical fiber with a diameter of $650 \mu \mathrm{m}$. The characteristics were measured by varying the pump energy by making use of a neutral density filter. The optical emission from the dyedoped optical fiber was excited by using $532 \mathrm{~nm}$ radiation from a frequency-doubled pulsed Nd:YAG laser (Quanta Ray DCR11) with a $10 \mathrm{~Hz}$ repetition rate and a $10 \mathrm{~ns}$ pulse width. The gain studies were done by transverse stripe illumination of the dyedoped fiber. The transverse pumping was done using the pump beam profile as a stripe obtained by employing a cylindrical lens of the appropriate focal length. The pump beam was shaped into a plane sheet $1 \mathrm{~cm}$ in length and $1 \cdot \mathrm{mm}$ in width by using a cylindrical lens. The spectral emission characteristics and the gain studies were carried out by collecting the fluorescence signal from the pumped fiber medium by using an optical fiber coupled to a monochromatorCCD system. To investigate the effect of feedback, a reflecting mirror was butt coupled to the other end of the optical fiber. Experiments were carried out with and without the mirror.

\section{B. Optical Fiber Amplifier}

The schematic of the experimental setup for the amplification studies is shown in Fig. 2. The pump beam $(532 \mathrm{~nm})$ was combined with the signal beam (at $585 \mathrm{~nm}$ ) both temporally as well as spatially by using the appropriate optical delay system and beam splitter. A photodetector of $1 \mathrm{~ns}$ response time (Newfocus-1621) along with a Tektronix digital storage oscilloscope $(500 \mathrm{MHz})$ was used to monitor the

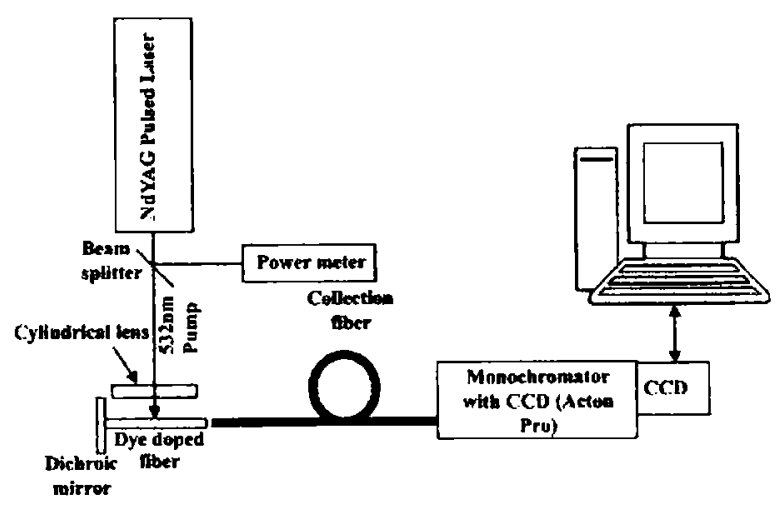

Fig. 1. Experimental setup for gain narrowing studies. 


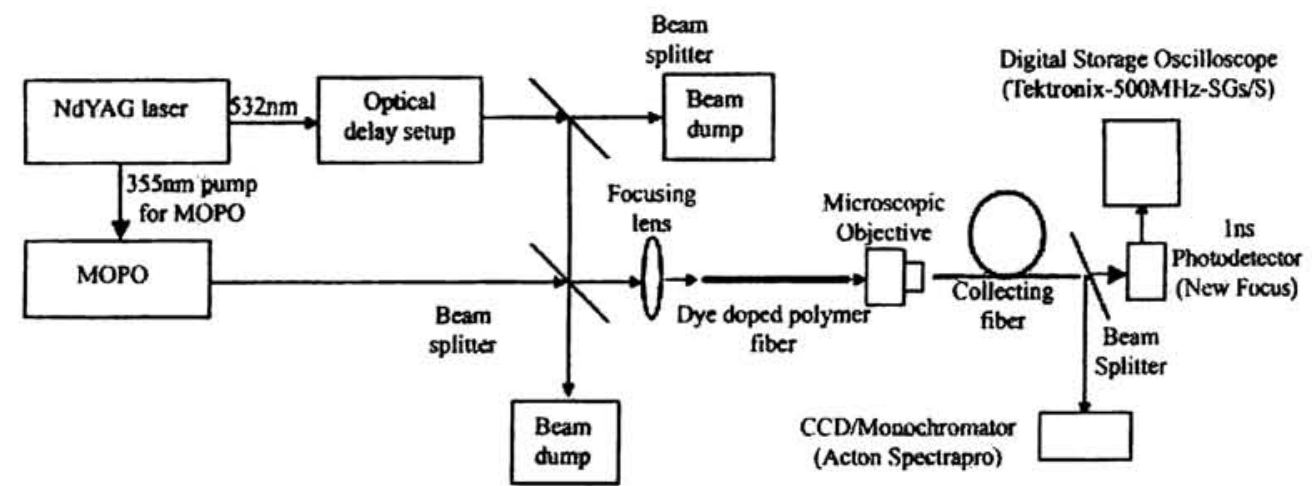

Fig. 2. Experimental setup for amplification studies of dye-doped fibers.

amplified output. A monochromator-CCD assembly (Acton Spectrapro) was used to study the spectral response of the output signal. The experiments were repeated for various pump powers and fiber lengths.

\section{Results and Discussion}

\section{A. Gain Medium}

Spectral line narrowing as a function of pump power is the signature of light amplification. Fluorescence emission spectra at various pump powers are illustrated in Fig. 3, which shows a clear line narrowing effect as pump power is increased.

Figure 4 is the plot of the FWHM of the spectral emission as a function of pump power. As is clear from the figure, spectral narrowing from 42 to $7 \mathrm{~nm}$ is observed when the pump power is increased to $6 \mathrm{~mJ}$ above which no further line sharpening is observed. As pump power is increased, spectral narrowing is observed owing to the fact that the number of modes for which the intensity is above the threshold decreases because of energy transfer from low-power modes to those lying near the emission peak. This

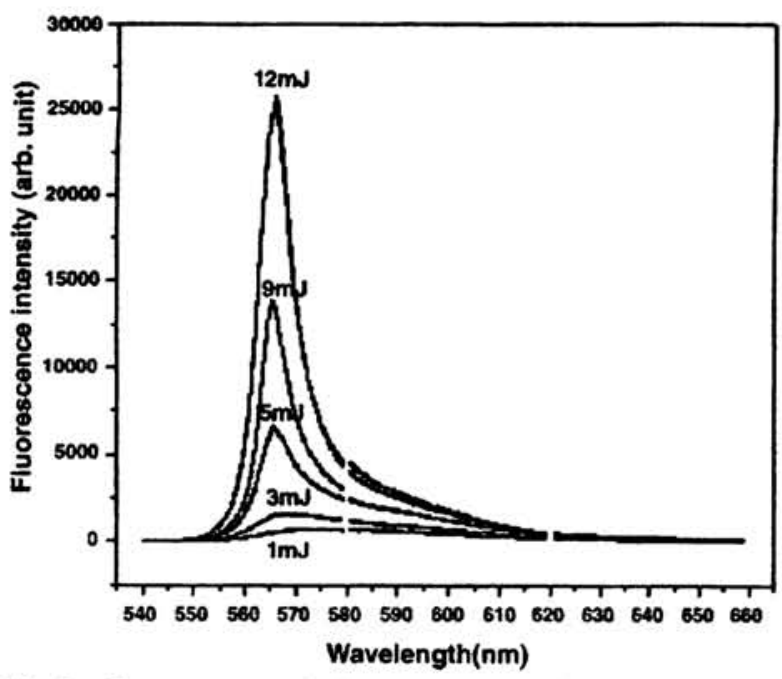

Fig. 3. Fluorescence emission spectra at various pump energies for a $5 \mathrm{~cm}$ long fiber. type of selective mode excitation around the line center with an increase in pump power is the signature of light amplification. Above a certain pump power, the number of modes with an intensity above the threshold is unchanged, leading to saturation of the linewidth.

To study the effect of the feedback mirror, a dichroic mirror was introduced at one end of the optical fiber. Figure 5 shows the emission spectra at $12 \mathrm{~mJ}$ with and without the mirror. It is clear from the figure that there is no significant change in FWHM However, one can see that the fluorescence signal strength is doubled. Note that the series of experments corresponding to Figs. 4 and 5 was done on the same fiber. The duration of pumping in the first set of experiments went above the bleaching level of the dye so that the results represented in Fig. 5 have a peak at a longer wavelength at a broader FWHM. Figure 6 is the plot exhibiting the variation of peak intensity as a function of pump energy. Above $6 \mathrm{~mJ}$ one can see a significant enhancement in the emission intensity with the introduction of the dichroic mirror, which is attributable to the feedback effect.

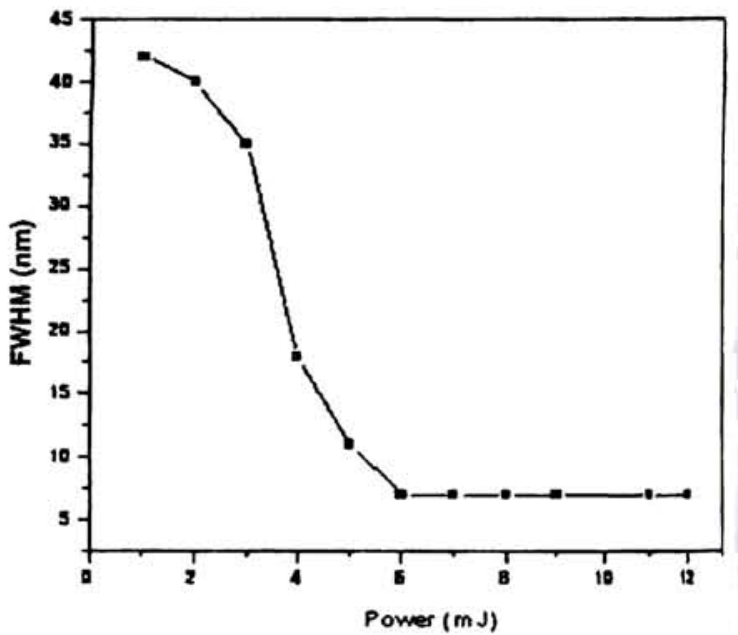

Fig. 4. Line narrowing with respect to an increase in pump en. ergy without using the reflecting mirror for a $5 \mathrm{~cm}$ long fiber. 


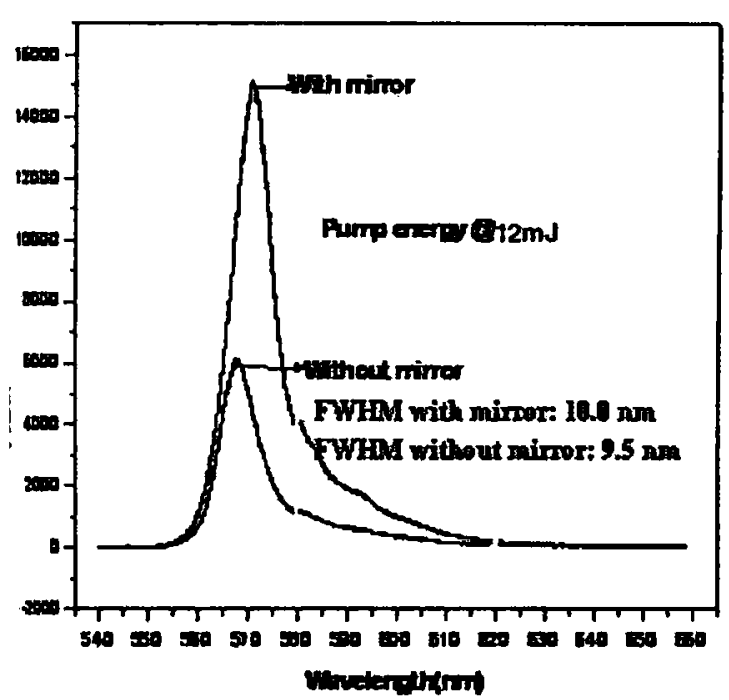

b. Plot of fluorescence emission with and without the reflectimirror at a pump energy of $12 \mathrm{~mJ}$. Fluorescence enhancement the mirror can be observed.

\section{Optical Fiber Amplifier}

it communication system one has to amplify weak "iteal signals. To check the efficiency of the dyebolymer optical fiber as an optical amplifier, feriments were carried out to measure the amplition factor by injecting a weak signal and meaing the output intensity with and without pump ver.

Ws can be seen in Fig. 7, there is a clear signal plification process in the presence of pump source, ch proves beyond a doubt that dye-doped optical $x$ can be used effectively as an amplifier in a commication link in the visible spectral region. Figure hows that a maximum gain of $18 \mathrm{~dB}$ is observed for ikngth of $8 \mathrm{~cm}$, which suggests that there is an pimum length to attain maximum amplification

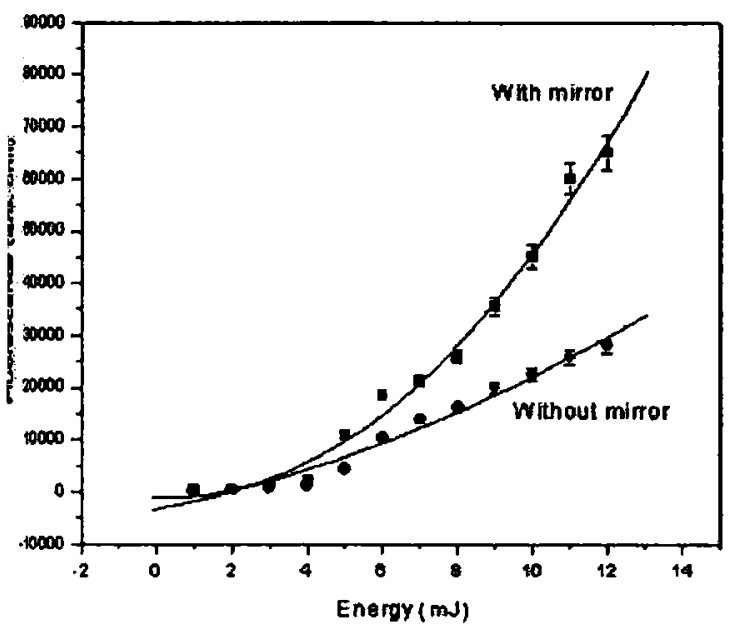

4. Plot of variation of peak intensity as a function of pump Aby. Above $6 \mathrm{~mJ}$ a significant enhancement in the emission with with the introduction of the dichroic mirror is observed.

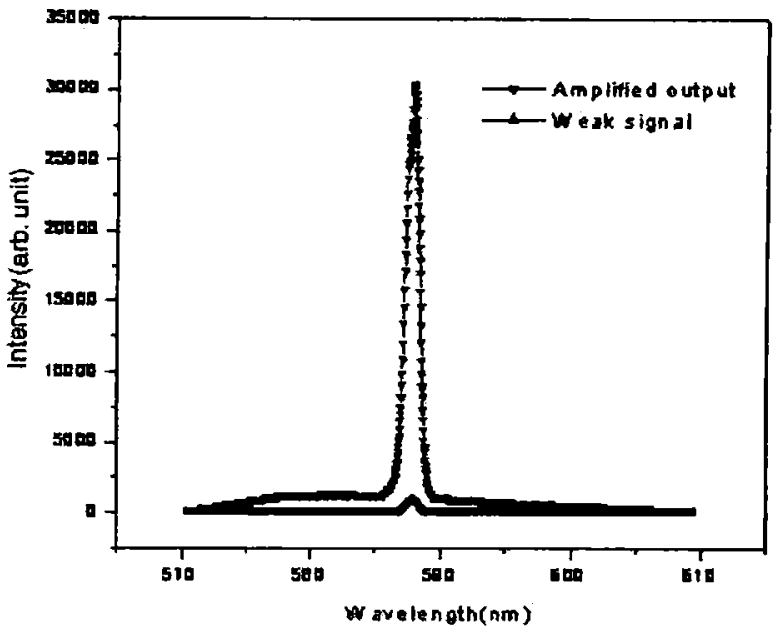

Fig. 7. Amplification of a weak signal in a dye-doped fiber $8 \mathrm{~cm}$ long.

(at $10^{-3}$ molar dye concentration). The fact that we can have a gain of $18 \mathrm{~dB}$ for a length of $8 \mathrm{~cm}$ is an advantage of dye-doped fiber in comparison with an erbium-doped fiber amplifier (EDFA), which needs a length of several meters to attain optimum gain.

Figure 9 shows that optimum gain is attained for an $8 \mathrm{~cm}$ long fiber as a function of pump power. The gain varies as a quadratic function of pump power. The pump power has to be limited below a certain level to minimize bleaching the dye molecules within the fiber. Figure 10 shows the intensity distribution of amplified signals at different wavelengths at a constant input signal power $(<0.2 \mathrm{~W}$ peak power $)$. The peak pump power was $1 \mathrm{~kW}$.

The intensity distribution shows enhanced signal amplification in the region at approximately $590 \mathrm{~nm}$. The spectrum shows maximum gain at $589 \mathrm{~nm}$ and another maximum at $640 \mathrm{~nm}$. The peak at approximately $589 \mathrm{~nm}$ is attributable to the char-

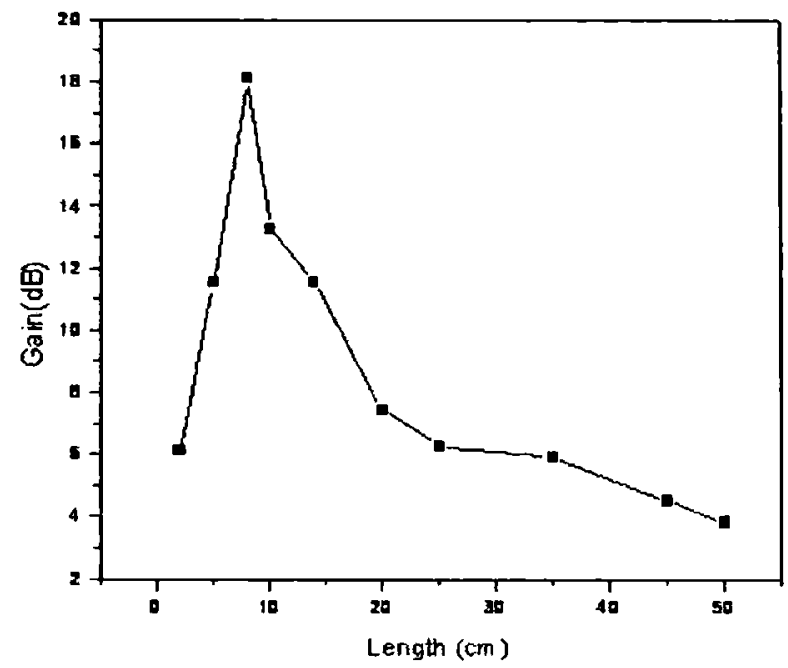

Fig. 8. Gain versus length of a dye-doped fiber amplifier. 


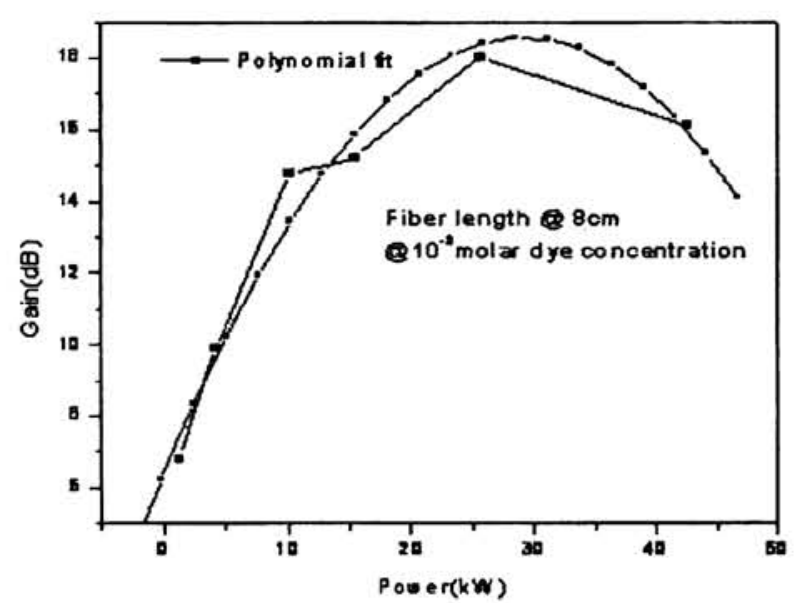

Fig. 9. Gain as a function of pump power.

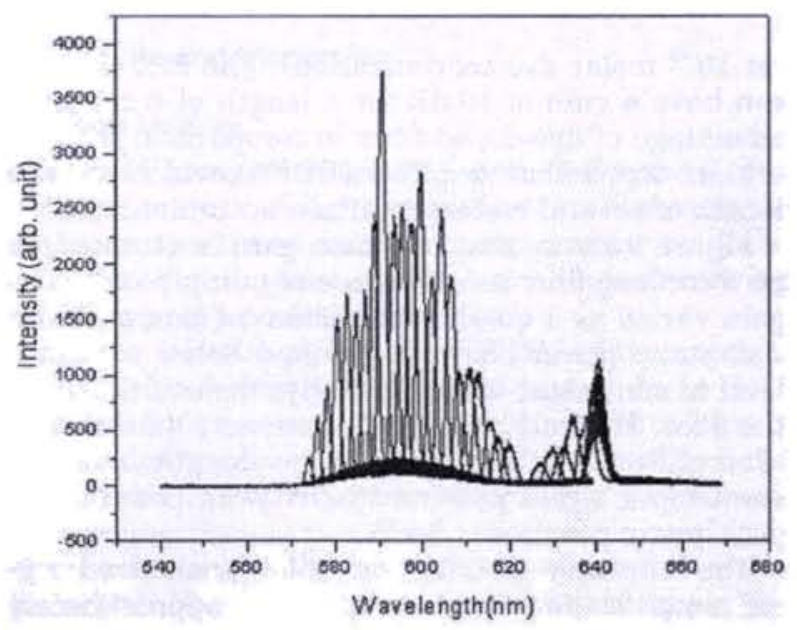

Fig. 10. Intensity distribution of various amplified signal wavelengths.

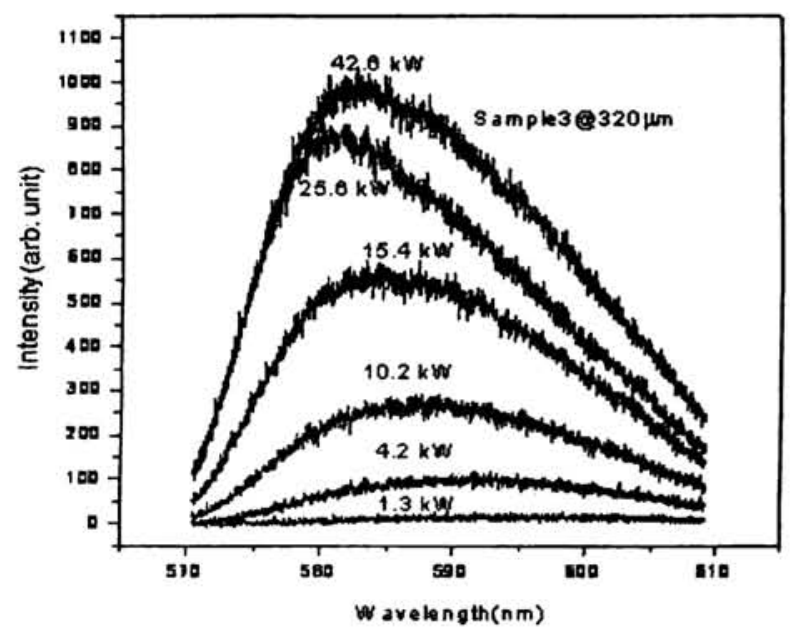

Fig. 11. Fluorescence blueshift observed as the pump power is increased.

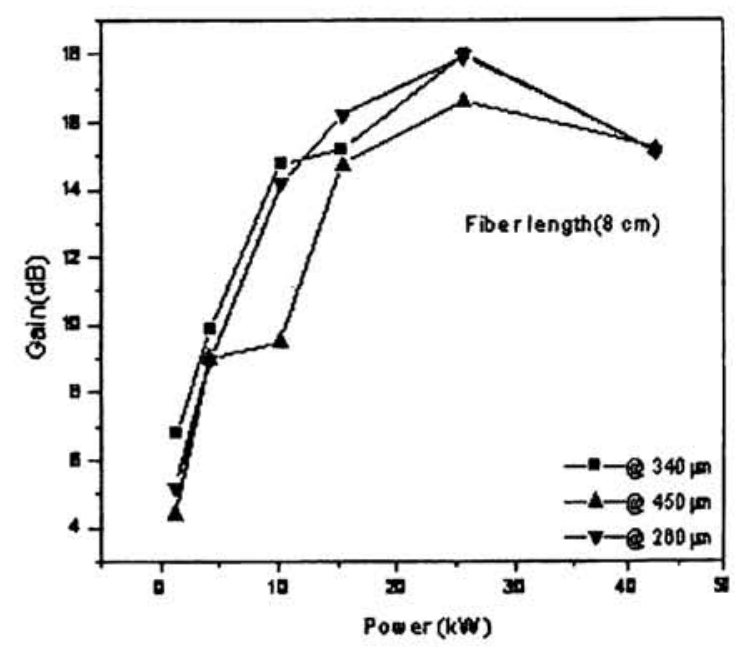

(a)

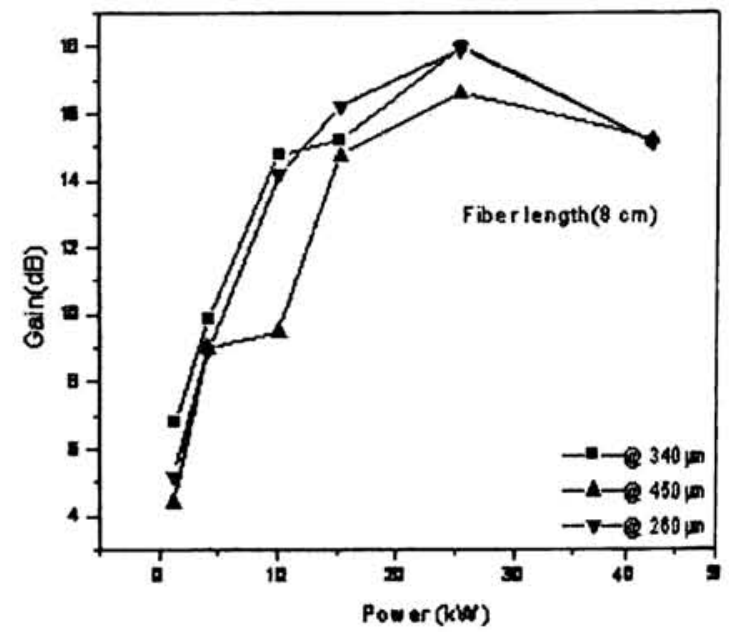

(b)

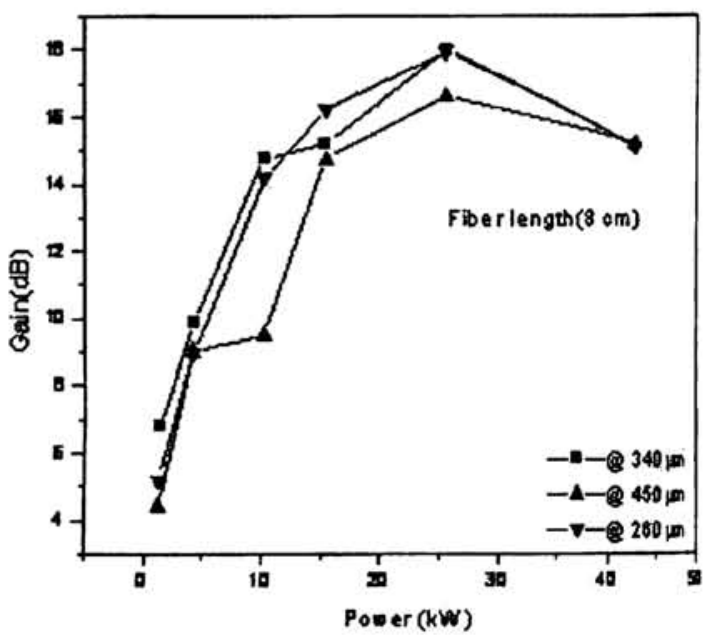

(c)

Fig. 12. Optical gain plot for various dye concentrations at various fiber diameters: (a) $1 \times 10^{-3} \mathrm{M}$ dye concentration. (b) $1 \times 10^{4}$ dye concentration, (c) $0.5 \times 10^{-4}$ dye concentration. 


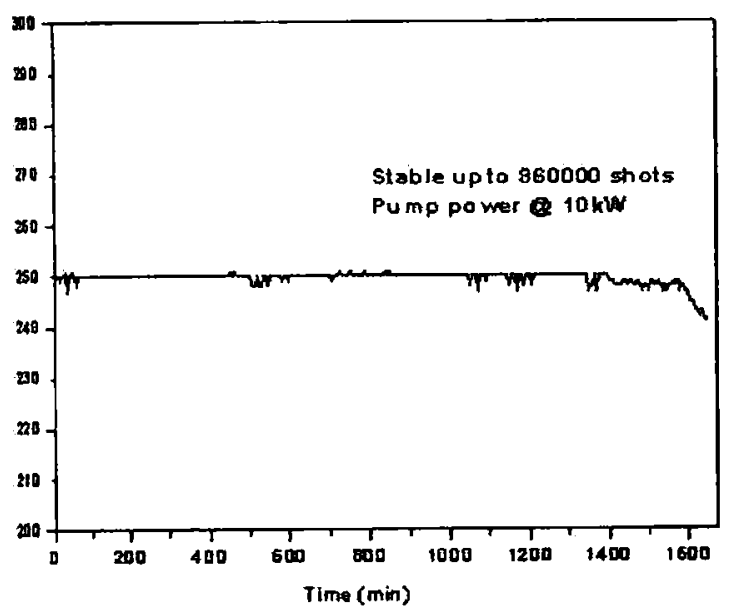

13. Graph showing fluorescence intensity versus time of vosure of dye-doped optical fiber to pump ( $532 \mathrm{~nm}$ ). The peak mp power was kept at $10 \mathrm{~kW}$.

seristic emission of Rhodamine 6G. The emission approximately $640 \mathrm{~nm}$ is attributed either to agegate formation or to emission followed by interstem crossing. As suggested by John and Pang ${ }^{19}$ nearest probable origin of the $640 \mathrm{~nm}$ emission is triplet-triplet transition. One can consider the $10 \mathrm{~nm}$ emission as an added advantage in the laser wission from the sample.

Pigure 11 depicts spectral dependence of fluoresme output as a function of pump power in the toped fiber with a diameter of $320 \mu \mathrm{m}$. There is a weshift in the emission spectra as the pump power is reased. The blueshift as a function of pump power is tributable to the transfer of mode energy to that of is shorter wavelength side, which has gotten a large in coefficient. However, the magnitude of this transtof energy gets saturated at higher pump power, and wis saturation is obtained as a redshift.

Figure 12 shows the variation of gain as a function ipump power as well as dye concentration. One of bebvious results is the enhancement of gain with concentration. A saturation of gain is observed at hor concentrations, and, moreover, the gain charderistics are sensitive to fiber diameter at lower mcentrations [Fig. 12(c)]. At higher concentrations ig. 12(a)] there is not much dependence of gain with diameter. This is because the increase in the meter is equivalent to the increase in the number imolecules involved in the excitation process. This id result in an effect equivalent to the concentramhancement resulting in gain saturation [Fig. 9ha). It is found that the fluorescence output from fiber was stable up to 960,000 shots of pump He without considerable fluorescence bleaching ia peak pump power of $10 \mathrm{~kW}$ at a laser repetition te of $10 \mathrm{~Hz}$ (Fig. 13).

\section{Conclusion}

bad quality dye-doped polymer optical fibers have drawn. The studies described in this paper show the performance and stability of Rhodamine 6Gdoped polymer optical fiber as an amplifying medium. An important observation is that there is an optimum length at which amplifier gain is at its maximum. For example, with $25 \mathrm{~kW}$ of pump power a maximum gain of $18 \mathrm{~dB}$ is attained for an $8 \mathrm{~cm}$ length of fiber. One of the important advantages to be noted is that the fiber length needed is very small unlike that for EDFA, which requires several meters. The polymer optical fiber amplifier working in current communication wavelengths can also be developed by using appropriate dyes or dopants. Studies in this direction are in progress.

M. Rajesh thanks the University Grants Commission, New Delhi, for his Center for Excellence in Lasers and Optoelectronic Sciences project fellowship. V. P. N. Nampoori acknowledges the financial assistance of the University Grants Commission, New Delhi, through a research award project. M. Sheeba and $\mathrm{K}$. Geetha thank the Council for Scientific and Industrial Research, New Delhi, and the University Grants Commission, New Delhi, respectively, for their research fellowships.

\section{References}

1. H. S. Nalwa, Polymer Optical Fibers (American Scientific, 2004).

2. G. P. Agrawal, Fiber Optic Communication Systems, 2nd ed. (Wiley-Interscience, 1997).

3. F. P. Kapron, D. B. Keck, and R. D. Maurer, "Radiation losses in glass optical fibers, ${ }^{\circ}$ Appl. Phys. Lett. 17, 423-425 (1970).

4. T. Kaino, K Jinguji, and S. Nara, "Low loss poly(methamethacrylate-d8) core optical fibers," Appl. Phys. Lett. 42, 567-569 (1983).

5. Y. Ohtsuka, T. Senga, and H. Yasuda, "Light-focusing plastic rod with low aberration," Appl. Phys. Lett. 25, 659-661 (1974).

6. Y. Ohtsuka, T. Sugano, and Y. Terao, "Studies on the lightfocusing plastic rod. 8: Copolymer rod of diethylene glycol bis(allyl carbonate) with methacrylic ester of fluorine containing alcohol, ", Appl. Opt. 20, 2319-2323 (1981).

7. Y. Koike, H. Hatanaka, and Y. Ohtsuka, "Studies on the light-focusing plastic rod. 17: Plastic GRIN rod lens prepared by photocopolymerization of a ternary monomer system," Appl. Opt. 23, 1779-1783 (1984).

8. W. Daum, J. Krauser, P. E. Zamzow, and O. Zeimann, POF. Polymer Optical Fibers for Data Communication, (Springer, 2002).

9. A. Costela, F. Florido, 1. Garcia-Moreno, R. Duchowicz, F. Amat-Guerri, J. M. Figuera, and R. Sastre, "Solid-state dye lasers based on copolymers of 2-hydroxyethyl methacrylate and methyl methacrylate doped with rhodamine 6G," Appl. Phys. B 60, 383-389 (1995).

10. G. D. Peng, P. L. Chu, Z. Xiong, T. W. Whitbread, and R. P. Chaplin, "Dye-doped step index polymer optical fiber for broadband optical amplification," J. Lightwave Technol. 14, 22152223 (1996)

11. K Kurki, T. Kobayashi, N. Imai, T. Tamura, S. Nishihara, Y. Nishizawa, A. Tagaya, and Y. Koike, "High-efficiency organic dye-doped polymer optical fiber lasers," Appl. Phys. Lett. 77, 331-333 (2000).

12. K. Kurki, T. Kobayashi, N. Imai, T. Tamura, Y. Koike, and Y. Okamoto, "Organic dye-doped polymer optical tiber lasers," Polym. Adv. Technol 11, 612-616 (2000).

13. H. Liang, Z. Zengchang, L. J. Xu, B. Chen, H. Zhao, Q. Zhang, 
and $H$. Ming, "Fabrication and amplification of Rhodamine B-doped step-index polymer optical fiber," J. Appl. Polym. Sci 93, 681-685 (2004).

14. A. Tagaya, S. Teramoto, E. Nihei, K. Sasaki, and Y. Koike, "High-power and high-gain organic dye-doped polymer optical fiber amplifiers: novel techniques for preparation and spectral investigation," Appl. Opt. 36, 572-578 (1997).

15. K. Geetha, M. Rajesh, V. P. N. Nampoori, C. P. G. Vallabhan, and P. Radhakrishnan, "Loss characterization in rhodamine 6G-doped polymer film waveguide by side illumination fluorescence," J. Opt. A Pure Appl. Opt. 6, 379-383 (2004).
16. K. H. Drexhage, "Structure and properties of laser dyes," in Dye Lasers, Vol. 1 of Springer Topics in Applied Physics, I.P Sheafer, ed. (Springer, 1990), Chap. 5, p. 155.

17. F. P. Sheafer, "Principles of dye laser operation," in Dye Lasent F. P. Sheafer, ed., Vol. 1 of Springer Topics in Applied Physios (Springer, 1990), Chap. 1, p. 1.

18. A. Tagaya, S. Teramoto, E. Nihei, K. Sasaki, and Y. Koite "High-power and high-gain organic dye-doped polymer optiol fiber amplifiers: novel techniques for prepration and spectral investigation, " Appl. Opt. 36, 572-578 (1997).

19. S. John and G. Pang, "Theory of lasing in a multiple-scattering medium," Phys. Rev. A 54, 3642-3652 (1996). 